\title{
Geriatrics in Clinical Practice and Beyond: Brain Health, the Sixth Vital Sign
}

Nannette Hoffman, MD ${ }^{1}$

\section{Abstract}

\section{Description}

Brain health in middle age and geriatric patients will take on greater importance for health care practitioners in our aging society. This article discusses various approaches clinicians can use for their patients to promote and improve brain health. These are generally the same lifestyle adoptions for cerebrovascular disease and cardiovascular disease prevention: weight management, exercise, and blood pressure and cholesterol lowering. Social stimulation and cognitive activities may also be important for the elderly.

\section{Keywords}

Alzheimer Disease; dementia; central nervous system diseases; geriatrics; early medical intervention; public health

\section{Introduction}

Ten thousand Americans turn 65 years old each day! By the end of the typical five-day work week 50,000 Americans have turned 65. Conversely the national population of children is at an all-time low of 24\%; in 1990 they were $40 \%$ of the American population. In 2018, those 65 years and over were $16 \%$ of the US population. ${ }^{2}$ The aging cohort is a growing segment of our populace for the long haul. These patients fill our emergency departments, urgent care centers, acute-care hospital beds and long-term care beds. Thus, it is imperative physicians and other health care providers continually broaden their knowledge to provide high quality elder care to delay or mitigate aging-related disability and cognitive impairment. Brain health and the specter of dementia is an important topic in this regard, not only for older adults but for the middle-aged too. As a Geriatrician I foresee brain health as the next vital sign, if not literally, then figuratively. This article's purpose is to provide a brief overview of contributors to dementia and how clinicians can modify them to delay the onset of this insidious disease.

\section{Background}

Remarkably, Americans 65 years and older with dementia dropped $24 \%$ between the years 2000 and 2012, according to an analysis of Health and Retirement Study data. ${ }^{3}$ This multifactorial decrease is due to increased life spans with better control of cardiovascular risk factors, like hyperlipidemia and hypertension, along with improved socioeconomic status and higher educational levels. The authors of the above analysis indicated that while higher educational levels appeared primarily responsible for the change, other unknown factors could have been involved.

Current thinking is higher educational levels and better education in youth boost cognitive reserves such that the better educated, healthier elder has greater cognitive compensatory mechanisms overcoming shrinkage of the hippocampus, the brain's memory center; also overcoming diffuse cerebral atrophy and neuronal death. ${ }^{4}$ In a prospective longitudinal study of nearly 320 subjects, participants with higher levels of occupational complexity tolerated smaller hippocampal volumes and greater whole-brain deterioration on Magnetic www.hcahealthcarejournal.com

(C) 2020 HCA Physician Services, Inc. d/b/a Emerald Medical Education
HCA Healthcare Journal of Medicine 
Resonance Imaging (MRI) studies, while performing at the same cognitive level as their peers with normal brain MRIs. ${ }^{5}$ In above study, after adjusting for confounding variables, those with less occupational complexity had decreased hippocampal volume and increased brain atrophy when participants were matched for cognitive function. These results suggested that in middle-aged persons at risk for dementia or Alzheimer's Disease, those with higher occupational complexity better tolerated aging neuropathology on neuroimaging and maintained a similar level of cognitive performance compared with those with less pathology.

Moreover, the study by Sisco et al. using longitudinal data from the Washington Heights-Inwood Columbia Aging Project showed significant ethnic and racial differences in dementia prevalence taking root in childhood and young adulthood. ${ }^{6}$ Approximately 4,000 older subjects were queried about their early education and had repeat cognitive testing over time. The authors found poorer educational quality was a predictor of lesser cognitive abilities in older age, more so for black study subjects than white subjects.

\section{Interventions}

There are several relevant approaches to potentially mitigate dementia and its progression in mid or late life: cognitive training, socialization, cardiovascular risk factor control and physical activity.

The Advanced Cognitive Training for Independent and Vital Elderly (ACTIVE) study illustrated that cognitive training in older people improved or maintained mental acuity, with training benefits evident a decade later. ${ }^{7}$ In this multi-site randomized clinical trial in six metropolitan areas, approximately 2,800 healthy adults ages 65 years and older participated in ten training sessions to strengthen memory, reasoning and speed of processing. At ten years, all groups showed declines from their baseline test performance. Analysis showed, as compared to controls, the intervention group had better maintenance of cognitive function at ten years such that $73.6 \%$ of reasoning-trained participants and $70.7 \%$ of speed-trained participants were performing at or above their respective cognitive ability compared to $61.7 \%$ and $48.8 \%$ respectively of control participants $(p<.01)$. Thus, participants who trained in reasoning and speed of processing experienced less cognitive decline. These findings support cognitive training might help older adults maintain mental abilities and stave off impairment. It is this author's view that this approach is less hazardous than the array of prescribed dementia-slowing medications of limited utility and with potential adverse side effects for older adults complaining of memory loss.

Higher vascular risk in middle age is associated with lower hippocampal brain volume and increased white matter intensities on cerebral imaging, harbingers of future dementia. In a British prospective cohort study, approximately 500 participants underwent magnetic resonance and amyloid positron emission tomography imaging at three time points (ages 36, 53 and 69 years). As Framingham Heart study cardiovascular risk scores increased with age so did the deterioration in brain volume and increase in white matter intensities. ${ }^{8}$ Importantly, there was no association of cardiovascular risk scores with amyloid deposits. At all points in time, worse scores were associated with smaller whole-brain volumes with largest effect sizes at age 36 years. In a randomized clinical trial, meta-analysis of blood pressure control in somewhat older adults, mean age 65 years, antihypertensive use was associated with lower incidences of dementia and cognitive impairment over four years. ${ }^{9}$ Thus, modifying vascular risk factors in younger and middle-aged adults potentially favorably impacts long term brain health as the population ages. ${ }^{10}$ Even older adults may benefit.

In another prospective cohort study of approximately 2000 community-dwelling older subjects who were cognitively intact at baseline and followed for at least five years, showed the risk of mild cognitive impairment was significantly reduced for subjects routinely engaging in multiple socially stimulating activities." Finally, exercise, such as walking, may delay dementia onset. In a large Japanese prospective observational five-year study of over 13,000 participants showed a significant inverse association $(p<0.001)$ between walking time and dementia incidence. ${ }^{12}$ 


\section{Conclusion}

Some health policy experts opine Alzheimer's Disease and related dementias are already a public health crisis with 5.7 million Americans living with a disease with no cure or disease altering treatment and one of the leading causes of death trailing cancer, heart disease and stroke..$^{13}$ Thus it is incumbent upon medical professionals, especially primary care clinicians, to proactively engage patients in controlling their hypertension and hyperlipidemia, improving physical activity and ensuring socialization and cognitive activities as early as possible, well before old age to promote brain health, the sixth vital sign.

\section{Acknowledgements}

I wish to thank Hale Toklu, PhD, MPharm, Division Director of Research, North Florida Regional Medical Center GME Program for her assistance with the manuscript.

\section{Conflicts of Interest}

The author declares she has no conflicts of interest.

Dr. Hoffman is an employee of North Florida Regional Medical Center, a hospital affiliated with the journal's publisher.

This research was supported (in whole or in part) by HCA Healthcare and/or an HCA Healthcare affiliated entity. The views expressed in this publication represent those of the author(s) and do not necessarily represent the official views of HCA Healthcare or any of its affiliated entities.

\section{Author Affiliation}

1. University of Central Florida/ HCA Healthcare North Florida Regional Medical Center, Gainesville, FL

\section{References}

1. Anne Tumlinson Innovations. A Call for Action: Creating an Optimal System of Brain Health Care in the United States. Brain Health Partnership. Published online 2019. https:// www.usagainstalzheimers.org/sites/default/ files/2019-04/BrainHealthOptimalSystemFINAL.pdf

2. U.S. - seniors as a percentage of the population 2050 | Statista. Published online 2019. https:// www.statista.com/statistics/457822/share-of- old-age-population-in-the-total-us-population/

3. Langa KM, Larson EB, Crimmins EM, et al. A Comparison of the Prevalence of Dementia in the United States in 2000 and 2012. JAMA Intern Med. 2017;177(1):51-58. https://doi.org/10.1001/ jamainternmed.2016.6807

4. Scommegna P, Mather M. Dementia Trends: Implications for an Aging America. Today's Research on Aging. 2017;(36):1-11.

5. Boots EA, Schultz SA, Almeida RP, et al. Occupational Complexity and Cognitive Reserve in a Middle-Aged Cohort at Risk for Alzheimer's Disease. Arch Clin Neuropsychol. 2015;30(7):634642. https://doi.org/10.1093/arclin/acv041

6. Sisco S, Gross AL, Shih RA, et al. The role of early-life educational quality and literacy in explaining racial disparities in cognition in late life. J Gerontol B Psychol Sci Soc Sci. 2015;70(4):557567. https://doi.org/10.1093/geronb/gbt133

7. Rebok GW, Ball K, Guey LT, et al. Ten-year effects of the advanced cognitive training for independent and vital elderly cognitive training trial on cognition and everyday functioning in older adults. J Am Geriatr Soc. 2014;62(1):16-24. https://doi.org/10.1111/jgs.12607

8. Lane CA, Barnes J, Nicholas JM, et al. Associations Between Vascular Risk Across Adulthood and Brain Pathology in Late Life: Evidence From a British Birth Cohort [published online ahead of print, 2019 Nov 4]. JAMA Neurol. 2019;77(2):1-9. https://doi.org/10.1001/jamaneurol.2019.3774

9. Hughes D, Judge C, Murphy R, et al. Association of Blood Pressure Lowering With Incident Dementia or Cognitive Impairment: A Systematic Review and Meta-analysis. JAMA. 2020;323(19):1934-1944. https://doi.org/10.1001/ jama.2020.4249

10. Seshadri S. Prevention of Dementia-Thinking Beyond the Age and Amyloid Boxes [published online ahead of print, 2019 Nov 4]. JAMA Neurol. 2019;1-2. https://doi.org/10.1001/jamaneurol.2019.3785

11. Krell-Roesch J, Syrjanen JA, Vassilaki M, et al. Quantity and quality of mental activities and the risk of incident mild cognitive impairment. Neurology. 2019;93(6):e548-e558. https://doi. org/10.1212/WNL.0000000000007897

12. Tomata Y, Zhang S, Sugawara Y, Tsuji I. Impact of time spent walking on incident dementia in elderly Japanese. Int J Geriatr Psychiatry. 2019;34(1):204-209. https://doi.org/10.1002/ gps.5011

13. Johnston M. Alzheimer's disease: A growing public health crisis. Alzheimer's Association: Northern California and Northern Nevada Chapter Blog. Published November 12, 2018. https:// www.alzheimersblog.org/2018/11/12/alzheimers-disease-public-health-issue/ 